\title{
S-cone discrimination in the presence of two adapting fields: data and model
}

\author{
Dingcai Cao \\ Visual Perception Laboratory, Department of Ophthalmology and Visual Sciences, The University of Illinois at Chicago, \\ Chicago, Illinois 60612, USA (dcao98@uic.edu)
}

Received October 3, 2013; revised November 25, 2013; accepted December 4, 2013; posted December 12, 2013 (Doc. ID 198616); published January 15, 2014

\begin{abstract}
This study investigated S-cone discrimination using a test annulus surrounded by an inner and outer adapting field with systematic manipulation of the adapting $l=\mathrm{L} /(\mathrm{L}+\mathrm{M})$ or $s=\mathrm{S} /(\mathrm{L}+\mathrm{M})$ chromaticities. The results showed that different adapting $l$ chromaticities altered S-cone discrimination for a high adapting $s$ chromaticity due to parvocellular input to the koniocellular pathway. In addition, S-cone discrimination was determined by the combined spectral signals arising from both adapting fields. The "white" adapting field or an adapting field with a different $l$ chromaticity from the other fields was more likely to have a stronger influence on discrimination thresholds. These results indicated that the two cardinal axes are not independent in S-cone discrimination, and the two adapting fields jointly contribute to S-cone discrimination through a cortical summation mechanism. (C) 2014 Optical Society of America

OCIS codes: $\quad$ (330.1720) Color vision; (330.4060) Vision modeling; (330.5510) Psychophysics; (330.7320)

Vision adaptation.

http://dx.doi.org/10.1364/JOSAA.31.000A65
\end{abstract}

\section{INTRODUCTION}

The early stage of chromatic processing is mediated by two anatomically distinct retinogeniculate pathways, the parvocellular (PC) and koniocellular (KC) pathways [1,2]. The PC pathway mediates the spectral opponency of $\mathrm{L}$ and $\mathrm{M}$ cones and contributes to red-green color vision. Four subgroups of PC cells have been identified, i.e., $+\mathrm{L} /-\mathrm{M},-\mathrm{L} /+\mathrm{M},+\mathrm{M} /-\mathrm{L}$, and $-\mathrm{M} /+\mathrm{L}$ cells. The $\mathrm{KC}$ pathway takes the difference between S-cone and L-, M-cone signals and contributes to blue-yellow color vision. The link between the two retinogeniculate pathways to psychophysics is well established using an equiluminant cone-based chromaticity space [3] or its derivative [4]. In a cone-based chromaticity space, the horizontal axis $\mathrm{L} /(\overline{\mathrm{L}}+\mathrm{M})$ represents the variation of relative $\mathrm{L}$-versus M-cone stimulation at equiluminance, while the vertical axis $\mathrm{S} /(\mathrm{L}+\mathrm{M})$ represents the variation in S-cone stimulation. Physiological recordings [5-7] indicate that $\mathrm{PC}$ and $\mathrm{KC}$ cells have preferred responses to $\mathrm{L} /(\mathrm{L}+\mathrm{M})$ and $\mathrm{S} /(\mathrm{L}+\mathrm{M})$ signals, respectively.

One fundamental question in color vision is whether the two cardinal axes in a cone-based chromaticity space are independent. Primate retinal ganglion cell recordings suggest there is negligible S-cone input to midget ganglion cells in the PC pathway and little $\mathrm{L} /(\mathrm{L}+\mathrm{M})$ input to the bistratified ganglion cells in the KC pathway [7]. However, S-OFF neurons in the macaque lateral geniculate nucleus (LGN) show L-cone signals oppose the combination of S- and M-cone signals [8]. In the primary visual cortex of primates, there are cells that prefer noncardinal color axes $[\underline{9}, \underline{10}]$. Psychophysical studies have also reported the interaction between the two cardinal axes, including chromatic contrast matching $[\underline{11}, \underline{12}]$, chromatic discrimination [13-18], color image classification [19], wavelength discrimination [20], chromatic induction [21], and color filling-in [22]. The existence of noncardinal color phenomena is thought to be mediated by higher-order cortical mechanisms (see review $[\underline{23}, \underline{24}]$ ).

Chromatic discrimination is a useful tool for understanding the mechanisms of color vision. It has been reported that L- or M-cone excitations from long-wavelength lights can have input to the S-cone pathway [18,25]. A recent discrimination study indicated that the two cardinal axes are not independent in $\mathrm{L} / \mathrm{M}$ discrimination [14]. However, typical chromatic discrimination experiments have been conducted in the presence of one adapting field. Chromatic discrimination in the presence of two or more adapting fields demonstrated different results from studies using one adapting field [26-29]. How multiple adapting fields contribute to chromatic discrimination is not well understood. In a recent study, L/M discrimination was measured in an annulus surrounded by inner and outer fields [30]. It was found that L/M discrimination thresholds were determined by contributions from both adapting fields. When none of the adapting fields were "white," the adapting field where the observer fixated always had a larger contribution to L/M discrimination. However, when one adapting field was "white," the "white" field was always weighted more heavily than the other adapting field in determining discrimination thresholds. These L/M discrimination results could not be accounted for by adaptation to the weighted average of two adapting chromaticities. Instead, the data were well described by a model based on the responses of midget ganglion cells in the PC pathway, which determined threshold by a summation of weighted PC-spectral responses between the test and each of the adapting fields separately. Since opponency between the test and two adapting fields is generated at the ganglion cell level and a nearly one-to-one projection from the ganglion cell level to the LGN 
[31], the summation of the two opponency signals needs to originate at a cortical site. In this sense, the L/M discrimination results suggest that a cortical mechanism determines the relative contributions from different adapting fields. It is unknown whether similar results can be obtained for S-cone discrimination. Therefore, the purpose of this study was to investigate whether the two cardinal axes were independent in S-cone discrimination and how two adapting fields contributed to determine discrimination thresholds.

\section{OBSERVERS}

The author, $D C$ (male, age 43 years), and another observer, $J G$ (male, age 20 years), participated in the study. Both have normal color vision (assessed by the Neitz OT anomaloscope) and hue discrimination (assessed by the Farnsworth-Munsell 100-hue test). Observer $J G$ was unaware of the purpose and design of the experiment at the time of data collection. All experimental procedures were approved by the University of Illinois at Chicago Institutional Review Board.

\section{EQUIPMENT}

The stimuli were generated using an iMac computer with a 10 bit video card to control a 17 -in. NEC CRT color monitor at a refresh rate of $75 \mathrm{~Hz}$. The CRT was well calibrated by measuring the spectral outputs of the red, green, and blue guns of the CRT using a Photo Research PR-670 Spectrophotometer. An International Light photometer IL-1700 measured the light outputs at 1024 steps to establish linearity of each gun and also verified the 10 bit resolution of the video card. The 10 bit video card can provide a resolution of 0.005 in $\mathrm{S} /(\mathrm{L}+\mathrm{M})$ resolution for an equiluminance stimulus, while the minimum threshold in the experiments was in the range of 0.03 in $S /(L+M)$, which was six times the $S /(L+M)$ resolution of the video card. In other words, the video card is sufficient for measuring S-cone discrimination.

\section{STIMULI}

The spatial configuration (Fig. 1A) included a $2^{\circ}$ wide annulus (inner diameter, $12^{\circ}$; outer diameter, $16^{\circ}$ ) surrounded by a circular inner $\left(12^{\circ}\right.$ in diameter) and an outer rectangular (full size of the CRT monitor) adapting field. The two adapting fields in the stimulus configuration did not have direct contact to avoid potential interactions between the two adapting fields that may affect chromatic discrimination in a complex way. A black cross $\left(0.14^{\circ}\right)$ served as the fixation point, set in the center of the inner field. A fan-shaped test patch with a central angle of 0.36 radians $\left(20^{\circ}\right)$ sat on the annulus, crossing the horizontal axis of the stimuli.

The luminance of the stimuli was $13.0 \mathrm{~cd} / \mathrm{m}^{2}$ (124.6 effective trolands (Tds) [32]). At this light level, it is possible that rods are active. However, rod contribution to the KC pathway was near zero at retinal illuminance higher than $20 \mathrm{Tds}[33,34]$. Heterochromatic flicker photometry (HFP, $12.5 \mathrm{~Hz}$ ) was used to establish equiluminance for each observer. Stimulus chromaticities were defined in a relative cone troland space [4] in which an equal-energy-spectrum light had a chromaticity of $l=\mathrm{L} /(\mathrm{L}+\mathrm{M})=0.665$ and $s=\mathrm{S} /(\mathrm{L}+\mathrm{M})=1.0$. The $s$ chromaticity of the annulus was chosen from the following: 0.6 , $0.8,1.0,1.2$, or 1.6 . The inner or outer field had $s$ chromaticity of $0.6,1.0$, or 1.6. The inner and outer fields may have had the same $l, s$ chromaticities depending on the design for different experiments. In Experiment 1, all three fields had the same $l$ chromaticities, i.e., $0.625,0.665$, or 0.705 , with equal inner and outer fields having the same $s$ chromaticity of $0.6,1.0$, or 1.6. In Experiment 2, the inner and outer fields had the same $s$ chromaticities, but one adapting field had a different $l$ chromaticity from the other adapting field and the annulus. In Experiment 3 , all three fields had the same $l$ chromaticities of 0.665 , and the inner and outer fields had different $s$ chromaticities. The chromaticities used in the study are shown in Fig. 1B.

\section{PROCEDURE}

At the beginning of a session, observers dark-adapted for $60 \mathrm{~s}$. A session consisted of the presentation of one pair of adapting fields with five annulus chromaticities. For each annulus chromaticity, observers adapted for $30 \mathrm{~s}$. S-cone discrimination was measured using a spatial two alternative forced choice
A

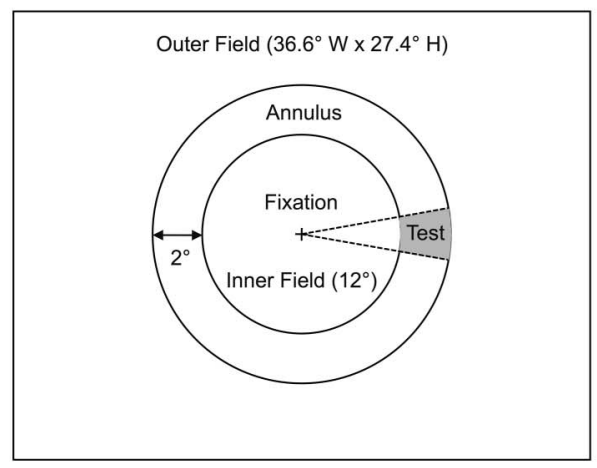

B

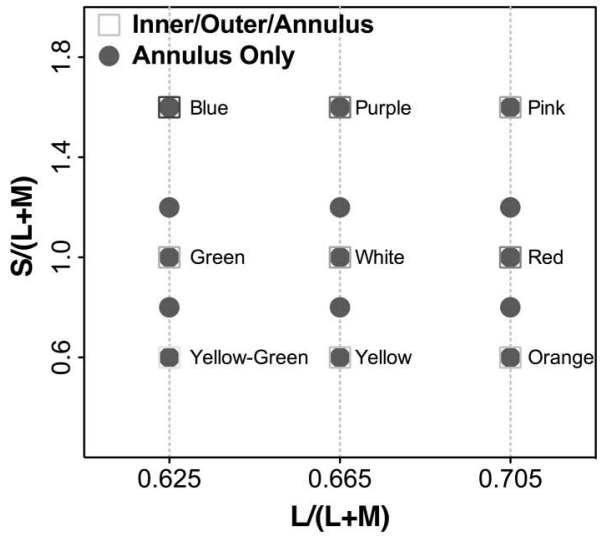

Fig. 1. A, stimulus spatial configuration; B, chromaticities. A, a $12^{\circ}$ (inner diameter) $-16^{\circ}$ (outer diameter) annulus was surrounded by an inner circular field and an outer rectangular field. A “+” $\left(0.14^{\circ}\right)$ served as fixation, which was located in the center of the inner field. The test patch was a section of the annulus with a central angle of $20^{\circ}$. B, stimulus chromaticities. The test annulus had an $s$ chromaticity of $0.6,0.8,1.0,1.2$, or 1.6 and $l$ chromaticity of $0.625,0.665$, or 0.705 . The chromaticities of the adapting fields were "yellow"-"green" $(l, s=0.625,0.6)$, "green" $(l, s=0.625,1.0)$, "blue" ( $l, s=0.625,1.6)$, "yellow" ( $l, s=0.665,0.6)$, "white" $(l, s=0.665,1.0)$, "purple" $(l, s=0.665,1.6)$, "orange" $(l, s=0.705,0.6)$, "red" $(l, s=0.705,1.0)$, and "pink" $(l, s=0.705,1.6)$. 
(2AFC) random double staircase procedure with one staircase for an increment and the other for a decrement in $s$ chromaticity. During each trial, the fan-shaped test patch was randomly presented to the left or right. The $s$ chromaticity change was presented in one cycle of a $1.5 \mathrm{~s}$ raised cosine window to minimize temporal transients or possible after-images. This stimulus presentation method has been used in other studies on S-cone discrimination [35]. Observers were instructed to maintain fixation during a trial and to identify the location of the test by pressing buttons on a gamepad sensed by the computer. The staircase procedure continued until 10 reversals occurred for each staircase. The last six reversals were averaged and were considered as the threshold estimate. The staircase procedure was then repeated for another annulus chromaticity. Each session lasted about $20 \mathrm{~min}$. Each session was repeated four to five times on different days.

\section{EXPERIMENT 1: S-CONE DISCRIMINATION WITH EQUAL INNER- OUTER ADAPTING $l$ AND $s$ CHROMATICITIES}

\section{A. Rationale and Design}

If there were no interaction between the $l$ and $s$ axes, the measured S-cone discrimination function would not be affected by varying $l$ chromaticities for the same $s$ adapting chromaticity. To test for interaction, the $l$ chromaticity for all three stimulus fields (the inner, outer adapting fields and annulus) was set as identical: $0.625,0.665$, or 0.705 . The $s$ chromaticities of the adapting fields were $0.6,1.0$, or 1.6 , leading to $9 s$ inner-outer field pairs [for $s=0.6$, "yellow green"-"yellow green" ( $l=0.625)$, "yellow"-“yellow" $(l=0.665)$, and "orange"-“orange" ( $l=0.705)$; for $s=1.0$, "green"-"green" $(l=0.625)$, "white"-"white" $(l=0.665)$, and "red"-"red" $(l=0.705)$; and for $s=1.6$, "blue"-"blue" $(l=0.625)$, "purple"-"purple" $(l=0.655)$, and "pink"-"pink" $(l=0.705)]$.

\section{B. S-Cone Discrimination Model}

\section{Model 1 for One Adapting Chromaticity Assuming}

\section{Independence between Two Cardinal Axes}

The model of S-cone pathway spectral processing is based on the spectral response of primate bistratified ganglion cells in the KC pathway [35, 36$]$. The model postulates two sites of adaptation in the $\mathrm{KC}$ pathway, i.e., first, a multiplicative gain in the cone receptors and, second, a negative feedback in the opponent pathway following spectral opponency of S-cones and the sum of L and M cones [37,38]. A schematic diagram of the model is shown in Fig. 2. In addition, the model assumes that the two cardinal axes are independent. That is, there is no PC-pathway input for differential L- and M-cone spectral processing to KC-pathway processing. The details of the mathematical implementation are as follows.

Step 1: Cone responses

The cone responses to a light of given L-, M- or S-cone trolands are given by

$$
\begin{aligned}
& R_{L}=L / l_{\max }, \\
& R_{M}=M / m_{\max }, \\
& R_{S}=S / s_{\max },
\end{aligned}
$$

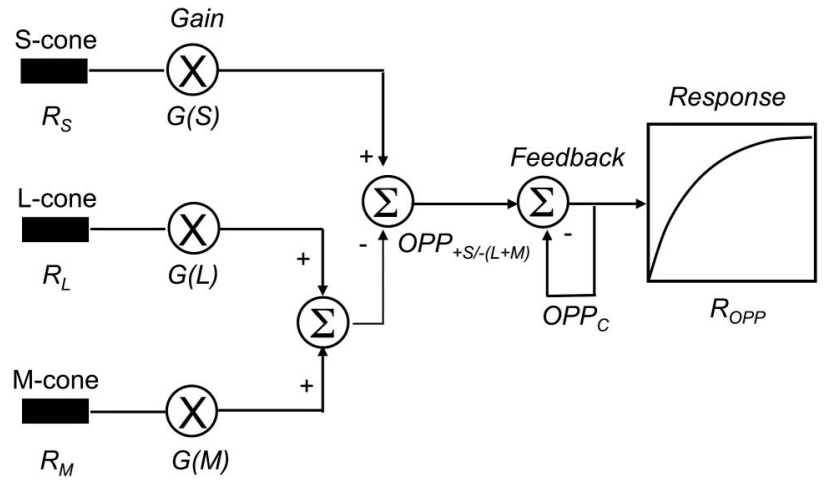

Fig. 2. Schematic diagram of the KC-spectral response model with one adapting chromaticity. See text for more details.

where $L, M$, and $S$ are cone trolands and $l_{\max }(=0.63721)$, $m_{\max }(=0.39242)$, and $s_{\max }(=1.6064)$ are maximal sensitivities of the Smith and Pokorny [39] cone fundamentals. The cone responses are subject to multiplicative sensitivity regulation (gain control):

$$
\begin{aligned}
& G\left(L_{A}\right)=1 /\left(1+k_{1} L_{A} / l_{\max }\right)^{k_{2}}, \\
& G\left(M_{A}\right)=1 /\left(1+k_{1} M_{A} / m_{\max }\right)^{k_{2}}, \\
& G\left(S_{A}\right)=1 /\left(1+k_{1} S_{A} / s_{\max }\right)^{k_{2},}
\end{aligned}
$$

where $L_{A}, M_{A}$, and $S_{A}$ are the cone trolands at the adapting chromaticity in the surround and $k_{1}$ and $k_{2}$ are constants. The value of $k_{1}$ is about $0.33 \mathrm{Td}$, and the value of $k_{2}$ is about 0.5 [40].

Step 2: S-cone pathway spectral opponency

The spectral opponent term for the $+\mathrm{S} /-(\mathrm{L}+\mathrm{M})$ cell is given by

$$
\begin{aligned}
\mathrm{OPP}_{+S /-(L+M)}= & S_{T} / s_{\max } G\left(S_{A}\right) \\
& -k_{3}\left[p L_{T} / l_{\max } G\left(L_{A}\right)\right. \\
& \left.+(1-p) M_{T} / m_{\max } G\left(M_{A}\right)\right]
\end{aligned}
$$

where $L_{T}, M_{T}$, and $S_{T}$ are cone trolands of the test chromaticity, $k_{3}$ is the surround strength of the opponent signal and has a value of 0.6 [35], and $p$ refers to the relative weight of $\mathrm{L}$ cones in the MC pathway for a Judd observer and has a value of 0.6189 . The spectral opponency signals are subject to subtractive feedback, which is determined by the strength of the opponent signal at the adapting chromaticity:

$$
\mathrm{OPP}_{C}=k_{5} \cdot\left(\mathrm{OPP}_{T}-k_{4} \mathrm{OPP}_{A}\right)
$$

where $\mathrm{OPP}_{C}$ is the spectral opponent signal to a chromaticity change, $C$, from a fixed adapting chromaticity, $A$, to a test chromaticity, $T$. $\mathrm{OPP}_{T}$ is the spectral opponent term at the test chromaticity, $\mathrm{OPP}_{A}$ represents the spectral opponent term at the adapting chromaticity, $k_{4}$ represents the subtractive feedback strength $\left(k_{4}=0.8\right.$ [35]), and $k_{5}$ (for the adapting $s$ chromaticity of 0.6 or $1.0, k_{5}=1$; for the adapting $s$ chromaticity of $1.6, k_{5}=0.2$ [35]). If $C=0$ (i.e., the test and adapting chromaticities are equal), then $\mathrm{OPP}_{T}$ is substituted by $\mathrm{OPP}_{A}$ in Eq. (ㅇ). 
Step 3: KC-pathway spectral contrast responses

The response of a spectral opponent cell to a chromaticity change, $C$, from a fixed adapting chromaticity, $A$, is

$$
R_{\mathrm{OPP}}=R_{\max } \cdot \mathrm{OPP}_{C} /\left(\mathrm{OPP}_{C}+\mathrm{SAT}\right),
$$

where $\mathrm{OPP}_{C}$ is a spectral opponent term in Eq. (8), $R_{\text {max }}$ is the maximum response, and SAT represents the value of the spectral opponency signal $\left(\mathrm{OPP}_{C}\right)$ for achieving half of the maximum response. Contrast gain is defined as $R_{\max } / \mathrm{SAT}$, the derivative of Eq. (9) at zero $\mathrm{OPP}_{C}$.

Step 4: S-cone discrimination threshold determination

Given that the criterion for discrimination $\delta$ is small relative to $R_{\max }$, the threshold of chromatic discrimination based on S-cone excitation can be deduced by the derivative of $R_{\mathrm{OPP}}$ in Eq. (9):

$$
\begin{aligned}
\log \left(\Delta S_{c}\right)= & \log \left(S_{\mathrm{th}}\right)-\log \left[G\left(S_{A}\right) / s_{\text {max }}\right] \\
& +\log \left[\left(\mathrm{OPP}_{C}+\mathrm{SAT}\right)^{2} / \mathrm{SAT}\right],
\end{aligned}
$$

where $S_{\text {th }}$ represents $\delta / R_{\max }$, a vertical scaling factor for sensitivity. In Eq. (10), S-cone discrimination is determined by three terms, the first $\left(S_{\text {th }}\right)$ related to the criterion and maximum response, the second $\left(G\left(S_{A}\right) / s_{\max }\right)$ related to S-cone gain control at the photoreceptor level, and the third related to the response to spectral opponency $\left(\mathrm{OPP}_{C}+\mathrm{SAT}\right)^{2} / \mathrm{SAT}$. There are two free parameters in this model, $S_{\text {th }}$ and SAT. This model has been successfully used to describe S-cone discrimination data in the presence of one adapting field [35].

\section{Model 2 for One Adapting Chromaticity Assuming PC Input to the KC Pathway}

The model presented in Eqs. (1)-(10) assumes there is no interaction between the two cardinal axes. To account for potential PC inputs to the KC pathway, Eq. (8) is revised to incorporate $\mathrm{PC}-$ spectral input to the $\mathrm{KC}$ pathway:

$$
\begin{aligned}
\mathrm{OPP}_{C}= & k_{5} \cdot\left[\left(\mathrm{OPP}_{T(\mathrm{KC})}+\alpha \mathrm{OPP}_{C(\mathrm{PC})}\right)\right. \\
& \left.-k_{4}\left(\mathrm{OPP}_{A(\mathrm{KC})}+\alpha \mathrm{OPP}_{C(\mathrm{PC})}\right)\right],
\end{aligned}
$$

where $\mathrm{OPP}_{C(\mathrm{KC})}$ and $\mathrm{OPP}_{A(\mathrm{KC})}$ represent the $\mathrm{KC}$-spectral signals from the test and adapting fields and are calculated by Eq. (7), and $\alpha$ is the weight of the PC-pathway spectral signal for $\mathrm{PC}$-input strength, $\mathrm{OPP}_{C(\mathrm{PC})}$. The linear combination of the $\mathrm{PC}$ - and $\mathrm{KC}$-spectral signals is consistent with the finding from color filling-in experiment [22]. The PC-pathway spectral signal for a $(+\mathrm{L} /-\mathrm{M})$ cell is computed as

$$
\begin{aligned}
\mathrm{OPP}_{C(+L /-M)}= & {\left[L_{T} / l_{\max } G\left(L_{A}\right)-0.8 M_{T} / m_{\max } G\left(M_{A}\right)\right] } \\
& -0.95\left[L_{A} / l_{\max } G\left(L_{A}\right)-0.8 M_{A} / m_{\max } G\left(M_{A}\right)\right] .
\end{aligned}
$$

The spectral signal for a $(+\mathrm{M} /-\mathrm{L})$ cell is calculated similarly to Eq. (12) by switching the order of L and M related terms. In Eq. (11), the larger value of the $(+\mathrm{L} /-\mathrm{M})$ or $(+\mathrm{M} /-\mathrm{L})$ was used for the PC-pathway spectral signal. The calculated response of a $(+\mathrm{L} /-\mathrm{M})$ cell at a retinal illuminance of $124.6 \mathrm{Tds}$ was $0.053,0.200$, and 0.348 for $l$ chromaticity of $0.625,0.665$, and 0.705 , respectively. Meanwhile, the calculated response of a $(+\mathrm{M} /-\mathrm{L})$ cell was $0.337,0.179$, and 0.02 for $l$ chromaticity of $0.625,0.665$, and 0.705 , respectively. Therefore, for the $l$ adapting chromaticity of 0.625 , the $(+\mathrm{M} /-\mathrm{L})$ response of 0.337 was used, while for the $l$ adapting chromaticity of 0.665 and 0.705 , the $(+\mathrm{L} /-\mathrm{M})$ responses of
0.200 and 0.348 were used. Equations (9) and (10) were applied to the value from Eq. (11) for fitting S-cone discrimination data with three free parameters, $S_{\mathrm{th}}$, SAT, and $\alpha$.

\section{Model Fits}

S-cone discrimination data with equivalent chromaticities in both adapting fields were fitted for both models using Eq. (10) with a common parameter value of SAT for the data with one adapting $l$ chromaticity for all three $s$ adapting chromaticities. The parameters were searched by minimizing the residual sum of squares.

\section{Results}

Figure 3 shows S-cone discrimination thresholds (in log S-cone Tds) as a function of annulus S-cone Tds with an adapting $s$ chromaticity of 0.6 (top panels), 1.0 (middle panels), or 1.6 (bottom panels) for the two observers. Each panel shows discrimination functions at the three $l$ adapting chromaticities for the same $s$ adapting chromaticity. The patterns for the adapting $s$ chromaticity of 0.6 and 1.0 were similar to those previously reported; that is, the minimum threshold occurred at the adapting chromaticity. The discrimination function for the adapting $s$ chromaticity of 1.6 was largely flat except with a low adapting $l$ chromaticity $(=0.625)$ and low annulus S-cone Td. The model assuming independence between the two cardinal axes failed to account for all of the data, particularly those at a high $s$ (1.0 or 1.6) or a low adapting $l$ chromaticity (Fig. 3A), with low or even negative $\mathrm{R}^{2}$ (proportion of variance explained) for those conditions (see Table 1 ; a negative $\mathrm{R}^{2}$ at $s=1.6$ indicates poor model performance because a model without considering PC input could never achieve a negative slope for adapting $s=1.6$ ). The model with PCpathway input to the KC pathway improved the fitting for those conditions (Fig. 3B; also see Table 1). For the $s$ adapting chromaticity of 0.6 , the input strength from the $(+\mathrm{L} /-\mathrm{M})$ signal to the KC pathway was zero or near zero (Fig. 4; also see Table 2) for both observers. For the $s$ adapting chromaticity of 1.0 or 1.6, the input strength decreased with increasing $l$ adapting chromaticity (Fig. 4). These results suggested that there was an input from $(+\mathrm{L} /-\mathrm{M})$ cells to the KC pathway in determining S-cone discrimination thresholds. In addition, the fitted value of SAT changed with different $l$ adapting chromaticities (Table 2), suggesting KC-pathway contrast gain was influenced by the strength of PC responses. Specifically, the value of SAT was the lowest for the $l$ adapting chromaticity of 0.665 with a weak PC response (the calculated response was 0.2 or 01.79 for the " $+\mathrm{L} / \mathrm{-M}$ " cells and “ $+\mathrm{M} / \mathrm{L}$ - cells, respectively) and then increased with a stronger $\mathrm{PC}$ response at a higher $l$ (mediated by " $+\mathrm{L} /$ - M" cells with the calculated response of 0.348 ) or lower $l$ (mediated by "+M/ - L" cells with the calculated response of 0.337 ) adapting chromaticity (Table 2). The modeling results indicated a reduction in KCpathway contrast gain with a stronger PC-pathway response.

\section{EXPERIMENT 2: S-CONE DISCRIMINATION WITH AN EQUAL INNER- OUTER ADAPTING $s$ CHROMATICITY BUT A DIFFERENT $l$ CHROMATICITY}

\section{A. Rationale and Design}

Experiment 1 demonstrated positive input from the $(+\mathrm{L} /-\mathrm{M})$ response to the $\mathrm{KC}$ pathway in S-cone discrimination. 

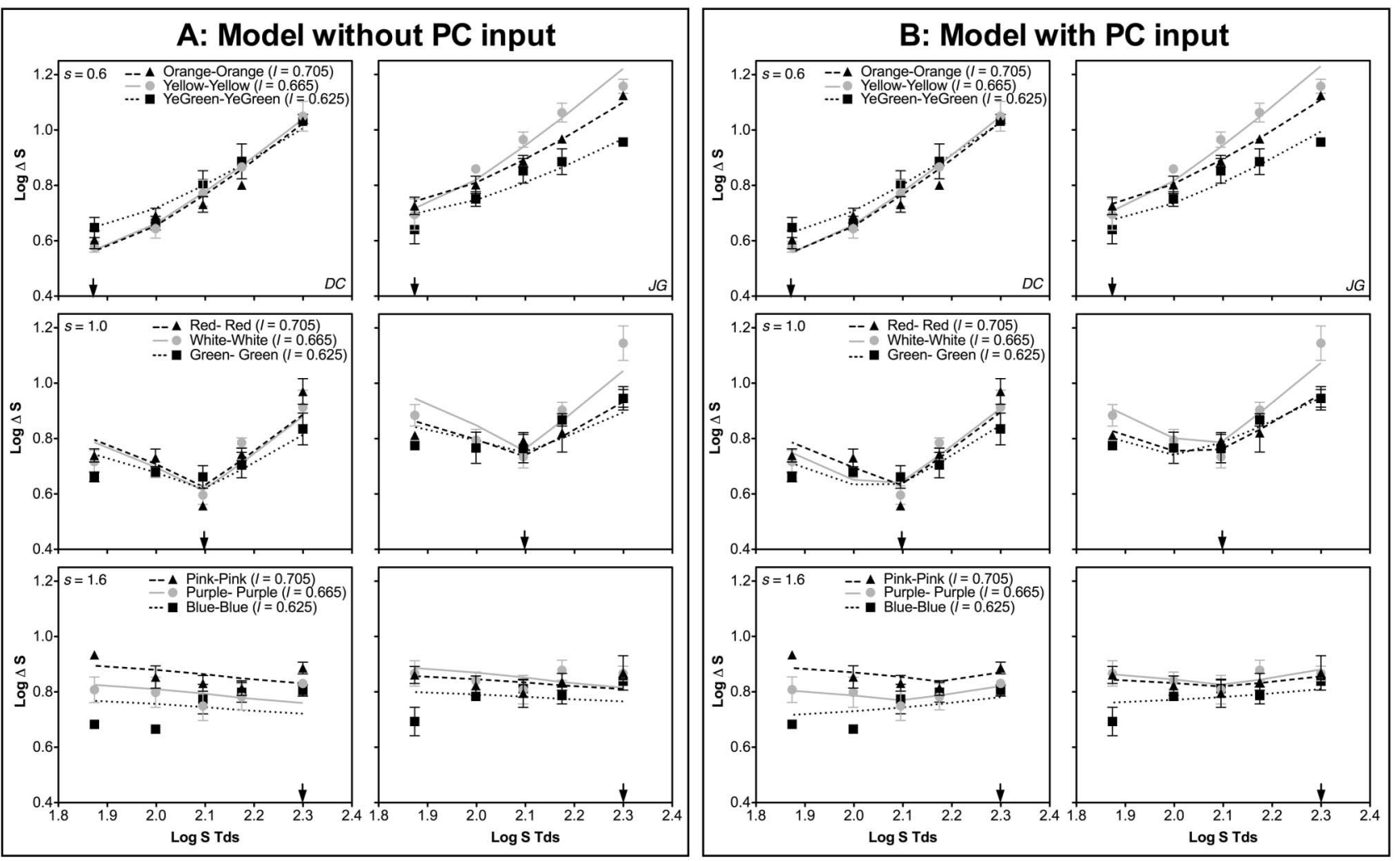

Fig. 3. S-cone discrimination thresholds from Experiment 1, expressed as $\log \Delta \mathrm{S}$ trolands, as a function of annulus log $\mathrm{S}$ trolands, for observers $D C$ and $J G$. Top row, adapting $s=0.6$; middle row, adapting $s=1.0$; bottom row, adapting $s=1.6$. Each panel shows the thresholds at three different adapting $l$ chromaticities of $0.625,0.665$, and 0.705 , with the adapting $s$ chromaticity indicated by an arrow. A, model fits assuming no PC-pathway input to the KC pathway (Model 1). B, model fits assuming PC-pathway input to the KC pathway (Model 2).

Table 1. $\mathbf{R}^{2}$ of the Fits from the Models without PC Input (Model 1) or with PC Input (Model 2) for Experiment 1

\begin{tabular}{|c|c|c|c|c|c|c|c|c|c|c|c|c|}
\hline \multirow[b]{3}{*}{$\begin{array}{l}\text { Adapting } \\
\text { Chromaticity }\end{array}$} & \multicolumn{6}{|c|}{ Obs. DC } & \multicolumn{6}{|c|}{ Obs. JG } \\
\hline & \multicolumn{2}{|c|}{$s=0.6$} & \multicolumn{2}{|c|}{$s=1.0$} & \multicolumn{2}{|c|}{$s=1.6$} & \multicolumn{2}{|c|}{$s=0.6$} & \multicolumn{2}{|c|}{$s=1.0$} & \multicolumn{2}{|c|}{$s=1.6$} \\
\hline & $\begin{array}{l}\text { No-PC } \\
\text { Input }\end{array}$ & $\begin{array}{l}\mathrm{PC} \\
\text { Input }\end{array}$ & $\begin{array}{l}\text { No-PC } \\
\text { Input }\end{array}$ & $\begin{array}{l}\mathrm{PC} \\
\text { Input }\end{array}$ & $\begin{array}{l}\text { No-PC } \\
\text { Input }\end{array}$ & $\begin{array}{l}\mathrm{PC} \\
\text { Input }\end{array}$ & $\begin{array}{l}\text { No-PC } \\
\text { Input }\end{array}$ & $\begin{array}{l}\mathrm{PC} \\
\text { Input }\end{array}$ & $\begin{array}{c}\text { No-PC } \\
\text { Input }\end{array}$ & $\begin{array}{l}\mathrm{PC} \\
\text { Input }\end{array}$ & $\begin{array}{c}\text { No-PC } \\
\text { Input }\end{array}$ & $\begin{array}{l}\mathrm{PC} \\
\text { Input }\end{array}$ \\
\hline$l=0.625$ & 0.960 & 0.977 & 0.566 & 0.752 & -0.569 & 0.500 & 0.910 & 0.910 & 0.523 & 0.906 & -0.465 & 0.451 \\
\hline$l=0.665$ & 0.996 & 0.955 & 0.789 & 0.897 & -1.030 & 0.815 & 0.945 & 0.935 & 0.810 & 0.914 & -1.419 & 0.462 \\
\hline$l=0.705$ & 0.930 & 0.923 & 0.814 & 0.827 & 0.161 & 0.568 & 0.990 & 0.997 & 0.637 & 0.909 & -0.604 & 0.673 \\
\hline
\end{tabular}

However, in Experiment 1, there was no $l$ contrast between the test and adapting fields. It is unknown whether S-cone discrimination is altered in the presence of an $l$ contrast. It is known that the S-cone pathway has poor spatial location information $[41,42]$. It is likely that adding an $l$ contrast will improve S-cone discrimination due to enhanced edge
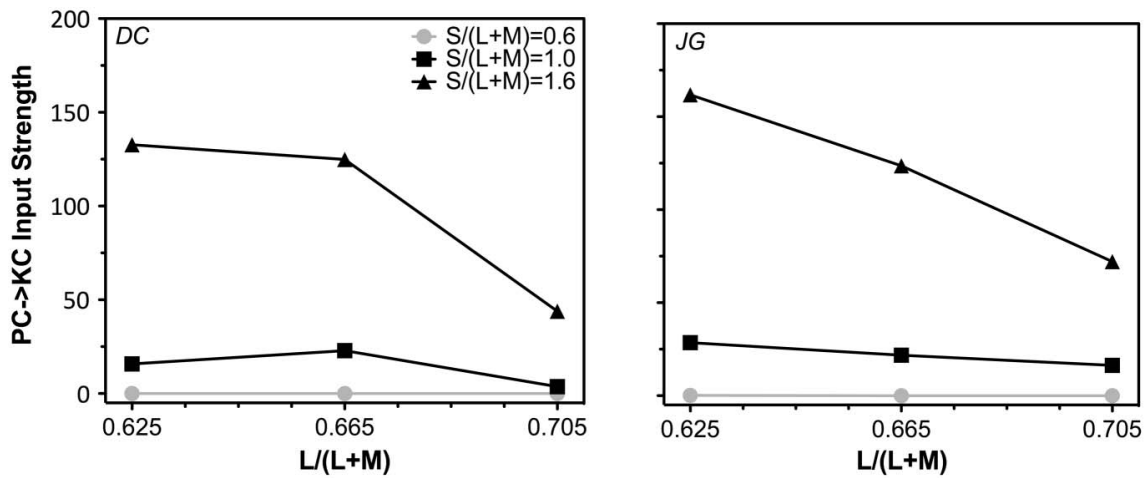

Fig. 4. Fitted input strength from PC-pathway spectral signals to the KC pathway. 


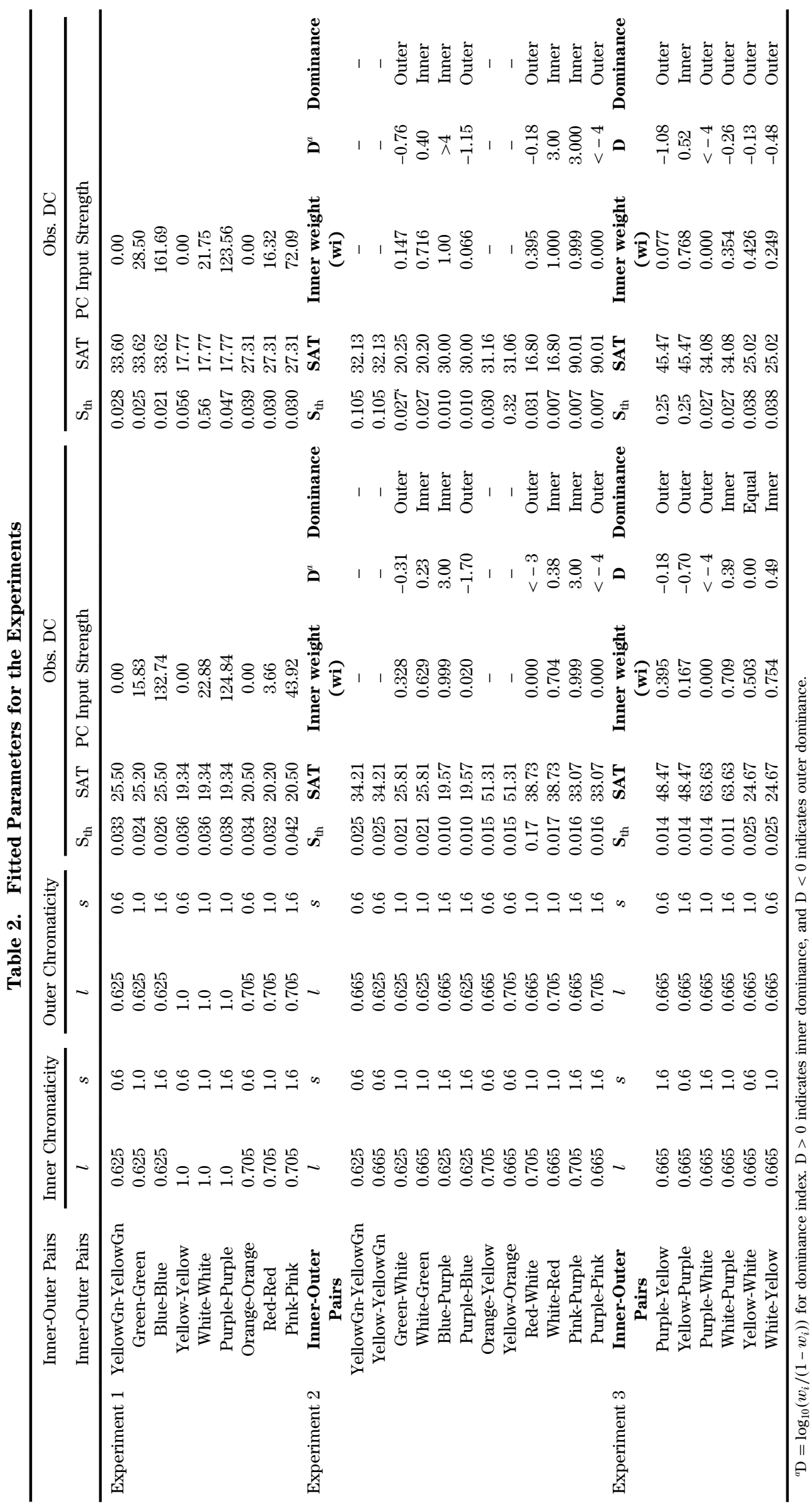


information [42, $\underline{43}]$. If edge information was important in Scone discrimination, the adapting field with a different $l$ chromaticity would play a more dominating role. To test this, Experiment 2 measured S-cone discrimination with the annulus and one adapting field having a fixed $l$ chromaticity of 0.665 and the other adapting field having an $l$ chromaticity of 0.625 or 0.705 . Therefore, the test annulus had $l$ contrast with one adapting field only. Four inner-outer field pairs were tested at each of three levels of $s$ [for $s=0.6$, two pairs with $l=0.625$ and 0.665 , and two pairs with $l=0.705$ and 0.665 ; for $s=1.0$, two pairs with $l=0.625$ and 0.665 , and two pairs with $l=$ 0.705 and 0.665 ; and for $s=1.6$, two pairs with $l=0.625$ and 0.665 , and two pairs with $l=0.705$ and 0.665 ; see Fig. $1 \mathrm{~B}]$.

\section{B. Models for Two Adapting Chromaticities}

To model S-cone discrimination with two adapting fields with different chromaticities ( $A_{1}$ with cone excitations of $L_{A 1}, M_{A 1}$, and $S_{A 1}$ and $A_{2}$ with cone excitations of $L_{A 2}, M_{A 2}$, and $S_{A 2}$ ), the model described in Eqs. (1)-(12) needed to be extended to account for the two adapting chromaticities. We have previously demonstrated that, with $\mathrm{L} / \mathrm{M}$ discrimination in the presence of two adapting fields [30], a retinal integration model assuming the spectral opponency signal generated by the test cone excitations and weighted average of cone excitations from the two adapting fields failed to account for the contributions from two adapting fields. Instead, a cortical integration model assuming the test field generates spectral opponency separately in relation to each of the two adapting fields, with the resulting opponency signals subsequently combined, was adopted. As the results in Experiment 1 showed PC-pathway input to the KC pathway, the spectral signal along one edge was computed using Eq. (11). Mathematically, spectral opponency signals are generated separately for the test and each of the two adapting fields,

$$
\begin{aligned}
\mathrm{OPP}_{C 1}= & k_{5} \cdot\left[\mathrm{OPP}_{T(\mathrm{KC})}+\alpha \mathrm{OPP}_{C 1(\mathrm{PC})}\right. \\
& \left.-k_{4}\left(\mathrm{OPP}_{A 1(\mathrm{KC})}+\alpha \mathrm{OPP}_{C 1(\mathrm{PC})}\right)\right], \\
\mathrm{OPP}_{C 2}= & k_{5} \cdot\left[\mathrm{OPP}_{T(\mathrm{KC})}+\alpha \mathrm{OPP}_{C 2(\mathrm{PC})}\right. \\
& \left.-k_{4}\left(\mathrm{OPP}_{A 2(\mathrm{KC})}+\alpha \mathrm{OPP}_{C 2(\mathrm{PC})}\right)\right],
\end{aligned}
$$

where $\mathrm{OPP}_{C 1}$ and $\mathrm{OPP}_{C 2}$ are opponency signals generated between the test and $A_{1}$ and between the test and $A_{2}$, respectively. Then the two opponency signals are pooled,

$$
\mathrm{OPP}_{C 1,2}=\left(\omega_{1} \mathrm{OPP}_{C 1}^{Q}+\omega_{2} \mathrm{OPP}_{C 2}^{Q}\right)^{1 / Q}
$$

where $\omega_{1}$ and $\omega_{2}\left(=1-\omega_{1}\right)$ are relative weights of the two opponency signals and $Q$ is the quick-pooling parameter. The fitted $Q$ values were between 0.65 and 1.348 for L/M discrimination [30]; therefore, for S-cone discrimination, $Q$ was set to be 1.0 to avoid over parameterization. The log transformation of the weight ratio, $D=\log \left(\omega_{1} / \omega_{2}\right)$, can be considered as an inner-outer dominance index, because if $D>0$, then the inner adapting field dominates the outer field in determining chromatic discrimination, while if $D<0$, then the outer field dominates. Finally, the pooled opponency signal is subject to a chromatic contrast response:

$$
R_{\mathrm{OPP} 1,2}=R_{\max } \cdot \mathrm{OPP}_{C 1,2} /\left(\mathrm{OPP}_{C 1,2}+\mathrm{SAT}\right) .
$$

The spectral responses along two edges $\left(\mathrm{OPP}_{C 1}\right.$ and $\left.\mathrm{OPP}_{C 2}\right)$ were derived from Model 2 with the same PC input strength and then fed into Eq. (15). There were four free parameters that included common parameters for both pairs, including $L_{\text {th }}$, SAT, and one weight parameter, $\omega_{1}$, for each pair.

\section{Results}

The S-cone discrimination functions for the adapting $l$ chromaticity combination of 0.625 and 0.665 are shown in Fig. 5A, and those for the adapting $l$ chromaticity combination of $0 . \overline{705}$ and 0.665 are shown in Fig. 5B. Each panel consists of two inner-outer pairs that swapped chromaticities in the inner and outer fields. The S-cone discrimination functions were similar whether the different $l$ chromaticity was set in the inner or outer field. Compared with the results in Experiment 1 with equal inner-outer chromaticities, an $l$ difference in one adapting field changed the S-cone discrimination function, suggesting both adapting fields may contribute to the measured threshold. Since for the $s$ adapting chromaticity of 0.6, there was no PC input based on Experiment 1's analysis (Fig. 4 and Table 2), there was no need to implement Eqs. (13)-(16) because two adapting fields resulted in the same KC-pathway spectral responses. However, the presence of a different $l$ chromaticity in one adapting field increased the fitted SAT values compared with the SAT values for the "yellow"-“yellow" pair in Experiment 1 (Table 2), suggesting an $l$ contrast along one edge reduced KC-pathway contrast gain. When the adapting $s$ chromaticity was 1.0 or 1.6 , the fitted SAT values were either equivalent to or larger than the SAT value for the "white"-"white" or "purple"-"purple" pairs in Experiment 1 (Table 2). The model fits based on Eqs. (13)(16) indicated that for the inner-outer pairs without a "white" adapting field, the adapting field with a different $l$ chromaticity from other fields always dominated the contribution, while for the pairs with a "white" adapting field, the "white" field always dominated the contribution (Table 2). The dominance pattern was similar to those observed in $\mathrm{L} / \mathrm{M}$ discrimination using the same stimulus configuration [30].

\section{EXPERIMENT 3: S-CONE DISCRIMINATION WITH AN EQUAL INNER- OUTER ADAPTING $l$ CHROMATICITY BUT A DIFFERENT $s$ CHROMATICITY}

\section{A. Rationale and Design}

Experiment 2 indicated that in the presence of an $l$ contrast along an edge, S-cone discrimination depended on the contributions from both adapting fields, with the field having a different $l$ chromaticity providing a dominating role. Experiment 3 further examined the inner-outer contributions but with the two adapting fields having different $s$ chromaticities. The $l$ chromaticities were set at 0.665 in all three fields. There were six inner-outer pairs, including "purple"-"yellow" ( $s=1.6$ and 0.6 , respectively), "yellow"-"purple" ( $s=0.6$ and 1.6, respectively), "purple"-"white" ( $s=1.6$ and 1.0, respectively), "white"-"purple" ( $s=1.0$ and 1.6, respectively), "yellow"-white" $(s=0.6)$ and 1.0, respectively), and "white"-"yellow" ( $s=1.0$ and 0.6 , respectively).

\section{B. Results}

Figure 6 shows the discrimination function in the presence of two $s$ adapting chromaticities [top row, "purple"-“yellow" 

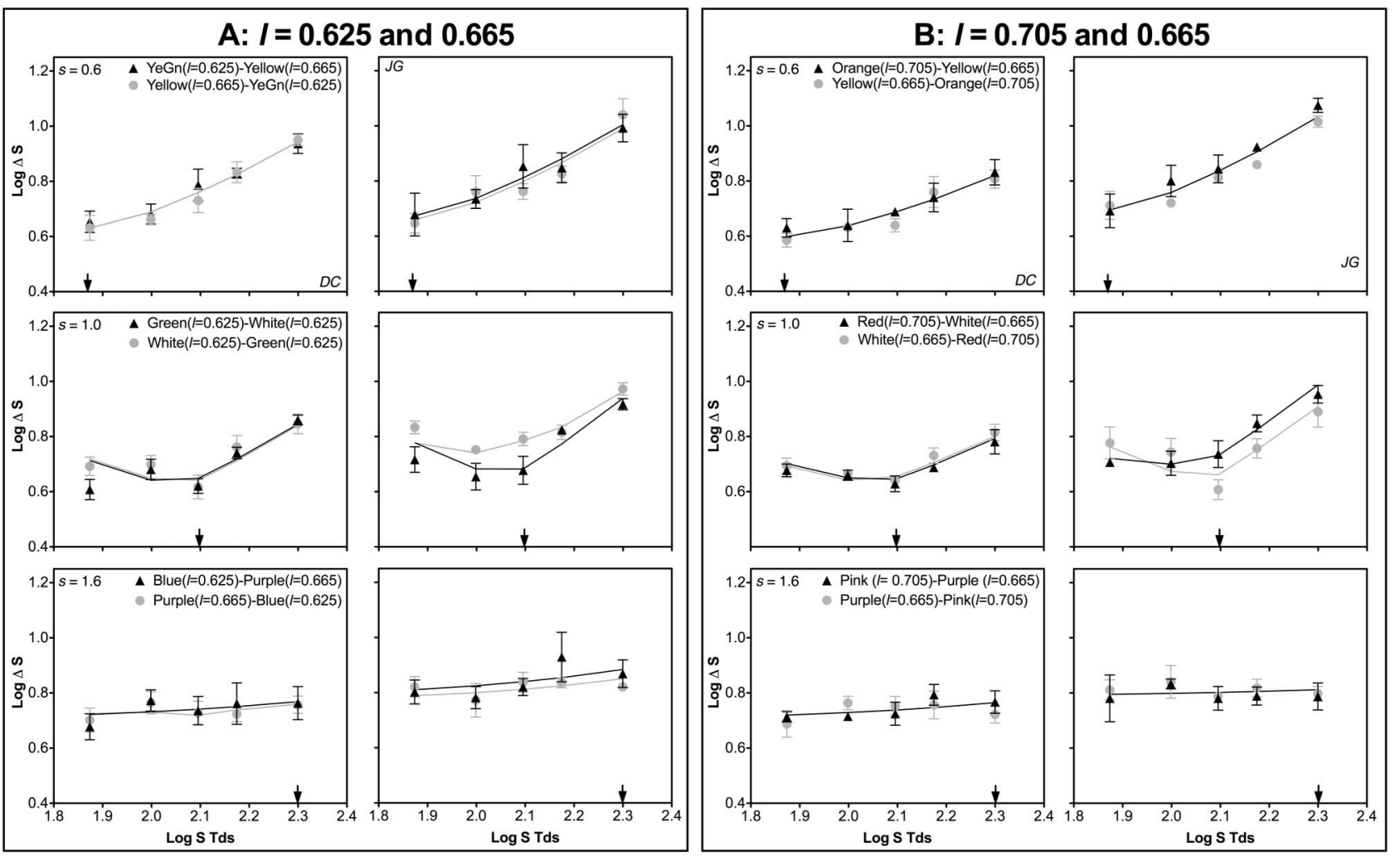

Fig. 5. S-cone discrimination thresholds from Experiment 2, with the two adapting fields having different $l$ but the same $s$ chromaticities. Top row, adapting $s=0.6$; middle row, adapting $s=1.0$; bottom row, adapting $s=1.6$. Each panel shows the data of two inner-outer pairs with chromaticities swapped between the inner and outer adapting fields. The solid lines are model fits based on integration of spectral signals from both adapting fields. A, the $l$ chromaticities in the two adapting fields were 0.625 and 0.665 . $\mathrm{B}$, the $l$ chromaticities in the two adapting fields were 0.705 and 0.665 .
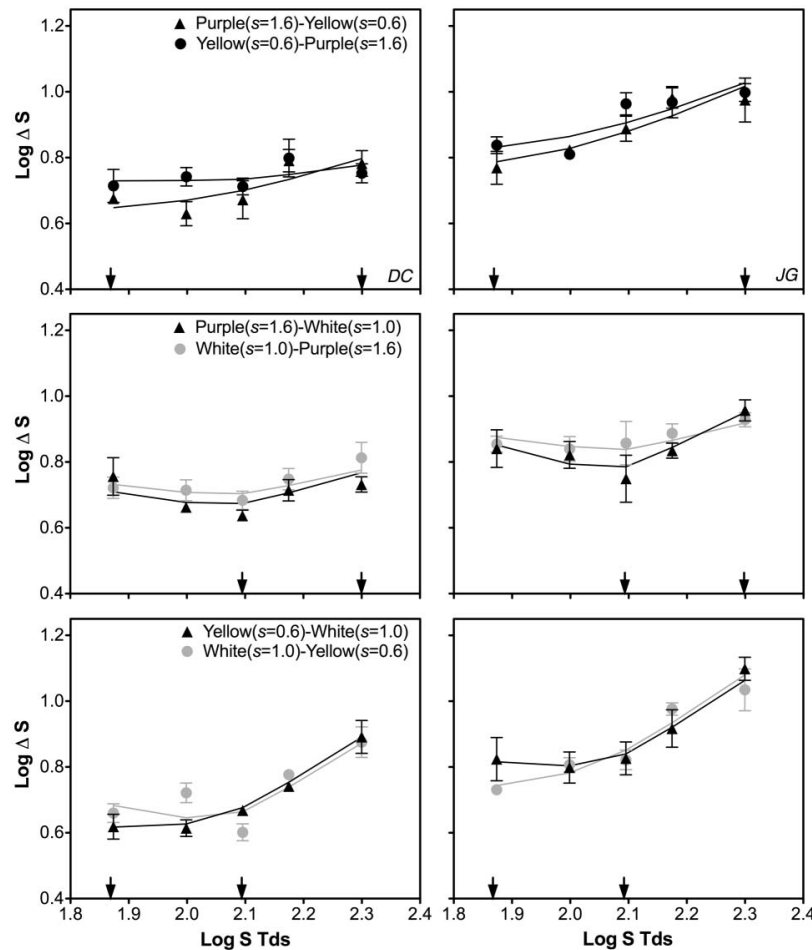

Fig. 6. S-cone discrimination thresholds from Experiment 3, with two adapting fields having different $s$ but the same $l$ chromaticities $(l=0.665)$. The arrows in each panel indicate the two adapting $s$ chromaticities. The solid lines are model fits based on integration of spectral signals from both adapting fields.
( $s=1.6$ and 0.6 ) and "yellow"-"purple" ( $s=0.6$ and 1.6) pairs; middle row, "purple"-"white" $(s=1.6$ and 1.0) and "white"-purple" ( $s=1.0$ and 1.6); bottom row, "yellow"-white" ( $s=0.6$ and 1.0) and "white"-"yellow" ( $s=1.0$ and 0.6 )]. The measured S-cone discrimination functions were different from the results in Experiment 1 with equal $l$ and $s$ chromaticities in both adapting fields, indicating a joint contribution from the two adapting fields. The model fits, by integrating spectral opponency signals along the two edges, could describe the data reasonably well $\left(\mathrm{R}^{2}=\right.$ 0.477-0.999 for observer DC or 0.712-0.975 for observer JG). The fitted weights of inner/outer fields indicated that the two observers had different inner-outer dominance patterns (Table 2). For observer DC, the "white" adapting field always dominated the other field if the pairs consisted of a "white" adapting field; otherwise the outer field dominated the inner field. For observer JG, the outer field always dominated if the pairs consisted of a "white" adapting field; otherwise the "yellow" field dominated.

\section{DISCUSSION}

This study demonstrated that S-cone discrimination in the presence of two adapting fields was determined by $\mathrm{KC}$ pathway processing between the test and both adapting fields. The modeling results indicated that fitting S-cone discrimination data required a PC-pathway input to the KC-pathway processing for a high $s$ adapting chromaticity of 1.0 or 1.6. In addition, the PC-pathway input strength decreased with increasing L- relative to M-cone excitations and a strong 
PC-pathway response increased KC-pathway contrast gain. This study also found that there was no PC-pathway input to the KC pathway for a low $s$ adapting chromaticity of 0.6 . These results are consistent with previous findings that the S-cone pathway receives L- versus M-cone opponency input from long-wavelength lights [18].

One fundamental question is where the neural locus is for combining the PC- and KC-spectral signals. McLellan and Eskew [18] modeled the KC-pathway spectral response by combining S-, L-, and M-cone excitations as

$$
Z=S-f(L, M)=S-10^{-k}(\alpha L+M) /[4|\gamma L-M|+1] .
$$

Their equation also included PC-opponency input to the $\mathrm{KC}$ pathway. In their equation, the L- versus M-opponency signal was thought to be a divisive inhibition or gain control, leading to a stronger KC-pathway spectral response with a larger value of the PC-opponency signal. Equation (17) implies a retinal original since the L- versus M-opponency signal affects the subtractive or inhibitory term only that feeds into S-bipolar cells in the retina to generate a KC-spectral opponency signal. In contrast, in Eq. (11) presented here, the PC-spectral signal was combined linearly with $\mathrm{KC}$-spectral signals arising from the test and adapting fields, with a larger PC signal leading to a larger combined response. Equation (11) suggests a postretinal combination of $\mathrm{PC}$ and $\mathrm{KC}$ signals. Given that the $\mathrm{PC}$ signal has the same sign as the $\mathrm{KC}$ signal and there is little $\mathrm{L} /(\mathrm{L}+\mathrm{M})$ input to the bistratified ganglion cell responses [7], the integration of $\mathrm{KC}$ and $\mathrm{PC}$ signals is most likely mediated by a postretinal mechanism.

In the presence of two adapting fields, the integration of KC-pathway spectral signals from two adapting fields must be due to a postretinal mechanism as well. The receptive fields for bistratified ganglion cells are not large enough to integrate the signals for the large stimuli used in this study. A nearly one-to-one projection from the ganglion cell level to the LGN [31] indicated that summing of the two spectral opponency signals from two adapting fields is not likely to be mediated by a retinal mechanism.

When one adapting field had a different $l$ chromaticity from the test and the other adapting field, the field with a different $l$ chromaticity or a "white" always dominated the contribution, similar to the findings on $\mathrm{L} / \mathrm{M}$ discrimination [30]. It has been suggested that the S-cone pathway has poor spatial location information $[\underline{41}, \underline{42}]$. Further, luminance contrast enhances blue-yellow signals in S-cone driven neurons in Macaque V1 through a nonlinear mechanism that combines nonopponent (luminance) and opponent signals [44]. There are cortical color-opponent neurons that receive S-cone as well as L- or Mcone inputs $[\underline{9}, \underline{45}, \underline{46}]$. Therefore, adding an $l$ contrast may change the response level of these cortical neurons to enhance the edge information and provide more influence to S-cone discrimination. In fact, in Experiment 3, when the two adapting fields differed only in $s$ chromaticities, the inner-outer field dominance patterns were not consistent among observers, further suggesting uncertain spatial information from KC-pathway processing. The effect of an $l$ contrast on S-cone discrimination also indicated the L-versus M-opponency signal is processed separately from the S-versus $(\mathrm{L}+\mathrm{M})$ signals at retinal processing and then combined at a later site such that the L-versus M-opponency signal could enhance spatial location information for S-cone discrimination. In this sense, Eq. (11) is more plausible than Eq. (17) to account for postretinal combination of PC- and KC-spectral signals.

Taken together, the study indicated PC-pathway input to the $\mathrm{KC}$ pathway in S-cone discrimination and a cortical mechanism integrating KC-pathway signals from two adapting fields to determine the discrimination threshold.

\section{ACKNOWLEDGMENTS}

This study was supported by NIH NEI grants R01 019651 (Cao, D.), UIC core grant for vision research P30-EY01792, and an Unrestricted Departmental Grant from the Research to Prevent Blindness. I thank Joseph Sukhbat Gansukh for his participation in data collection and his assistance in preparing the figures. I also thank Drs. Margaret Lutze and Xiaohua Zhuang for their comments on this manuscript.

\section{REFERENCES}

1. D. M. Dacey, "Parallel pathways for spectral coding in primate retina," Annu. Rev. Neurosci. 23, 743-775 (2000).

2. B. B. Lee, "Visual pathways and psychophysical channels in the primate," J. Physiol. 589, 41-47 (2011).

3. D. I. A. MacLeod and R. M. Boynton, "Chromaticity diagram showing cone excitation by stimuli of equal luminance," J. Opt. Soc. Am. 69, 1183-1185 (1979).

4. V. C. Smith and J. Pokorny, "The design and use of a cone chromaticity space," Color Res. Appl. 21, 375-383 (1996).

5. A. M. Derrington, J. Krauskopf, and P. Lennie, "Chromatic mechanisms in lateral geniculate nucleus of macaque," J. Physiol. 357, 241-265 (1984).

6. B. B. Lee, J. Pokorny, V. C. Smith, P. R. Martin, and A. Valberg, "Luminance and chromatic modulation sensitivity of macaque ganglion cells and human observers," J. Opt. Soc. Am. A 7, 2223-2236 (1990).

7. H. Sun, H. E. Smithson, Q. Zaidi, and B. B. Lee, "Specificity of cone inputs to macaque retinal ganglion cells," J. Neurophysiol. 95, 837-849 (2006).

8. C. Tailby, S. G. Solomon, and P. Lennie, "Functional asymmetries in visual pathways carrying S-cone signals in macaque," J. Neurosci. 28, 4078-4087 (2008).

9. B. R. Conway, "Spatial structure of cone inputs to color cells in alert macaque primary visual cortex (V-1)," J. Neurosci. 21, 2768-2783 (2001).

10. B. R. Conway, "Color vision, cones, and color-coding in the cortex," Neuroscientist 15, 274-290 (2009).

11. M. A. Webster and J. D. Mollon, "Changes in colour appearance following post-receptoral adaptation," Nature 349, 235-238 (1991).

12. M. A. Webster and J. D. Mollon, "The influence of contrast adaptation on color appearance," Vis. Res. 34, 1993-2020 (1994).

13. M. V. Danilova and J. Mollon, "Parafoveal color discrimination: a chromaticity locus of enhanced discrimination," J. Vis. 10(1): 1-9 (2010).

14. M. V. Danilova and J. D. Mollon, "Cardinal axes are not independent in color discrimination," J. Opt. Soc. Am. A 29, A157-A164 (2012).

15. R. T. Eskew, J. R. Newton, and F. Giulianini, "Chromatic detection and discrimination analyzed by a Bayesian classifier," Vis. Res. 41, 893-909 (2001).

16. J. Krauskopf, D. R. Williams, M. B. Mandler, and A. M. Brown "Higher order color mechanisms," Vis. Res. 26, 23-32 (1986).

17. J. R. Newton and R. T. Eskew, "Chromatic detection and discrimination in the periphery: a postreceptoral loss of color sensitivity," Vis. Neurosci. 20, 511-521 (2003).

18. J. S. McLellan and R. T. Eskew, "ON and OFF S-cone pathways have different long-wave cone inputs,” Vis. Res. 40, 2449-2465 (2000).

19. T. Hansen and K. R. Gegenfurtner, "Classification images for chromatic signal detection,” J. Opt. Soc. Am. A 22, 2081-2089 (2005). 
20. K. T. Mullen and J. J. Kulikowski, "Wavelength discrimination at detection threshold," J. Opt. Soc. Am. A 7, 733-742 (1990).

21. D. C. Cao and S. K. Shevell, "Chromatic assimilation: spread light or neural mechanism?" Vis. Res. 45, 1031-1045 (2005).

22. X. Zhuang and D. Cao, "Contrast magnitude and polarity effects on color filling-in along cardinal color axes," J. Vis. 13(7): 19 (2013).

23. R. T. Eskew, "Higher order color mechanisms: a critical review," Vis. Res. 49, 2686-2704 (2009).

24. K. R. Gegenfurtner, "Cortical mechanisms of colour vision," Nat. Rev. Neurosci. 4, 563-572 (2003)

25. J. D. Mollon and P. G. Polden, "An anomaly in the response of the eye to light of short wavelengths," Phil. Trans. R. Soc. B 278, 207-240 (1977).

26. M. D. Fairchild and P. Lennie, "Chromatic adaptation to natural and incandescent illuminants," Vis. Res. 32, 20772085 (1992)

27. A. Li and P. Lennie, "Mechanisms underlying segmentation of colored textures," Vis. Res. 37, 83-97 (1997).

28. T. Hansen, M. Giesel, and K. R. Gegenfurtner, "Chromatic discrimination of natural objects," J. Vis. 8(1):2 (2008).

29. M. Giesel, T. Hansen, and K. R. Gegenfurtner, "The discrimination of chromatic textures,” J. Vis. 9(9), 11 (2009).

30. D. Cao and Y. Lu, "Chromatic discrimination: differential contributions from two adapting fields," J. Opt. Soc. Am. A 29, A1-A9 (2012).

31. P. D. Spear, C. B. Y. Kim, A. Ahmad, and B. W. Tom, "Relationship between numbers of retinal ganglion cells and lateral geniculate neurons in the rhesus monkey,” Vis. Neurosci. 13, 199-203 (1996).

32. Y. LeGrand, Light, Colour and Vision, 2nd ed. (Chapman \& Hall, 1968), pp. 1-564.

33. D. Cao, J. Pokorny, and V. C. Smith, "Matching rod percepts with cone stimuli," Vis. Res. 45, 2119-2128 (2005).
34. D. Cao, J. Pokorny, V. C. Smith, and A. J. Zele, "Rod contributions to color perception: linear with rod contrast," Vis. Res. 48, 2586-2592 (2008).

35. D. Cao, A. J. Zele, V. C. Smith, and J. Pokorny, "S-cone discrimination for stimuli with spatial and temporal chromatic contrast," Vis. Neurosci. 25, 349-354 (2008)

36. J. Pokorny and V. C. Smith, "Chromatic discrimination," in The Visual Neuroscience, L. M. Chalupa and J. S. Werner, eds. (Massachusetts Institute of Technology, 2004), pp. 908-923.

37. E. N. J. Pugh and J. D. Mollon, "A theory of the $\pi-1$ and $\pi-3$ color mechanisms of Stiles," Vis. Res. 19, 293-312 (1979).

38. Q. Zaidi, A. Shapiro, and D. Hood, "The effect of adaptation on the differential sensitivity of the S-cone color system," Vis. Res. 32, 1297-1318 (1992).

39. V. C. Smith and J. Pokorny, "Spectral sensitivity of the foveal cone photopigments between 400 and 500 nm," Vis. Res. 15, 161-171 (1975).

40. E. Miyahara, V. C. Smith, and J. Pokorny, "How surrounds affect chromaticity discrimination,” J. Opt. Soc. Am. A 10, 545-553 (1993).

41. B. W. Tansley and R. M. Boynton, "A line, not a space, represents visual distinctness of borders formed by different colors," Science 191, 954-957 (1976).

42. B. W. Tansley and R. M. Boynton, "Chromatic border perception the role of red- and green-sensitive cones," Vis. Res. 18, 683-697 (1978).

43. J. Mollon, "Seeing colour," in Colour: Art \& Science (1995), pp. $127-150$

44. G. D. Horwitz, E. Chichilnisky, and T. D. Albright, "Blue-yellow signals are enhanced by spatiotemporal luminance contrast in macaque V1," J. Neurophysiol. 93, 2263-2278 (2005).

45. G. D. Horwitz, E. Chichilnisky, and T. D. Albright, "Cone inputs to simple and complex cells in V1 of awake macaque," J. Neurophysiol. 97, 3070-3081 (2007).

46. B. R. Conway and M. S. Livingstone, "Spatial and temporal properties of cone signals in alert macaque primary visual cortex," J. Neurosci. 26, 10826-10846 (2006) 